\title{
Magnetostatic dipolar domain-wall pinning in chains of permalloy triangular rings
}

\author{
P. Vavassori, ${ }^{1,2,3}$ D. Bisero, ${ }^{2,3}$ V. Bonanni, ${ }^{2}$ A. Busato, ${ }^{2}$ M. Grimsditch, ${ }^{4}$ K. M. Lebecki, ${ }^{5}$ V. Metlushko, ${ }^{6}$ and B. Ilic ${ }^{7}$ \\ 'CIC nanoGUNE Consolider, E-20009 San Sebastian, Spain \\ ${ }^{2}$ CNISM and Dipartimento di Fisica, Università di Ferrara, I-44I00 Ferrara, Italy \\ ${ }^{3}$ CNR-INFM National Research Center S3, via Campi 213a, 1-41100 Modena, Italy \\ ${ }^{4}$ Materials Science Division, Argonne National Laboratory, Argonne, Mlinois 60439, USA \\ ${ }^{5}$ Institute of Physics, Polish Academy of Sciences, 02-668 Warsaw, Poland \\ ${ }^{6}$ Department of Electrical and Computer Engineering. University of Illinois at Chicago, Chicago, Illinois 60607, USA \\ ${ }^{7}$ Cornell Nanofabrication Facility, School of Applied and Engineering Physics, Cornell University, Ithaca, New York 14853, USA
}

\begin{abstract}
In a combined experimental and numerical study, we investigated the details of the motion and pinning of domain walls in isolated and interacting permalloy triangular rings (side $2 \mu \mathrm{m}$, width $250 \mathrm{~nm}$, and thickness $25 \mathrm{~nm}$ ). To induce interaction between the rings, they were arranged either in vertical chains with an apex of each triangle in proximity to the edge center of the triangle above it or in horizontal chains where the proximity is between the adjacent corners of the triangles. Using longitudinal and diffraction magneto-optic Kerr effects, magnetic force microscopy, and micromagnetic simulations, we determined the field dependence of the spin structure in the rings. In all cases the remnant state of each ring is an "onion" state characterized by two domain walls—one head to head the other tail to tail—pinned at the apexes. In isolated rings the magnetization reversal occurs between two onion states via the formation of an intermediate vortex state, which arises from the motion and annihilation of the two domain walls. In the case of the horizontal chains the reversal mechanism is unchanged except that the dipolar interaction affects the field range in which the rings are in the vortex state. In the case of vertical chains an additional intermediate state is observed during reversal. The new state involves a domain wall pinned at the center of the edge that is in close proximity to the apex of its neighbor. We show that the domain-wall motion in this last case can be modeled by a triple potential well. Because the new state requires that a domain wall be pinned at the neighboring apex, our observations can be viewed as a very elementary form of magnetic logic.
\end{abstract}

PACS number(s): 75.75.-a, 75.60.Ch, 75.60.Jk, 78.20.Ls

\section{INTRODUCTION}

Manipulation of magnetic domain walls (DWs) in nanostructures has recently become the focus of intense research due to its great potential for the application to spintronics and also because of the basic physics involved in the phenomenon. Crucial for applications is the possibility to induce a controlled and reproducible DW displacement by either using magnetic fields or injecting polarized currents. Magnetic nanorings are a particularly apt geometry to such investigations. If the effects of magnetocrystalline anisotropy are negligible, only the geometry of the ring determines the microscopic spin structure of the magnetic states. The effects of geometrical constrictions, such as notches and corners, on DWs in nanorings, ${ }^{1-3}$ as well as the effects of magnetostatic coupling on the collective switching ${ }^{4-7}$ and on the DW structure $^{8}$ in closely packed arrays of interacting rings have been intensively investigated. Of particular interest has recently become the study of the possibility of controlling the domain-wall motion in nanowires using magnetostatic interactions. For example, the pinning of domain walls by localized magnetic fields generated by nanomagnets placed in the vicinity of nanowires can be applied to the so-called "magnetic domain-wall logic" (Ref. 9) in order to implement logic gate operations.

In an earlier work we have studied the magnetization reversal in permalloy (Py) square and triangular rings and we observed that head-to-head DWs can be positioned at se- lected corners and moved between corners by applying a field along a particular direction. ${ }^{10.11}$ These structures enable the intentional design of clockwise and anticlockwise magnetic vortex ground states. ${ }^{10,11}$ Here we present the results of a combined experimental and numerical study with which we determined the details of the pinning and the displacement of DWs in isolated and interacting Py triangular rings. The small width ( $250 \mathrm{~nm}$ in the present study) of the ring segments is a crucial parameter in order to have stable magnetization configurations determined by a controllable fieldinduced displacement of DWs between corners. Indeed, in the case of much wider equilateral Fe triangular rings (segments' width larger than $1 \mu \mathrm{m}$ ) a previous detailed magneto-optical Kerr effect investigation showed a much more complicated remagnetization process occurring through the formation of complex domains structures rather than DWs displacement. ${ }^{12}$ The simplicity and reproducibility of the magnetization configurations in the structures investigated here allowed us to detect and investigate subtleties related to the DWs motion within the three sides of the triangles. The domain-wall propagation within a given branch was also found to depend on the magnetic state of neighboring triangles thereby providing yet another route via which the magnetic ground state can be manipulated. Based on the results presented here, we envision that this type of magnetic rings could be used as input and output logic gates for pure magnetic cellular automata systems. ${ }^{13}$ 
a)
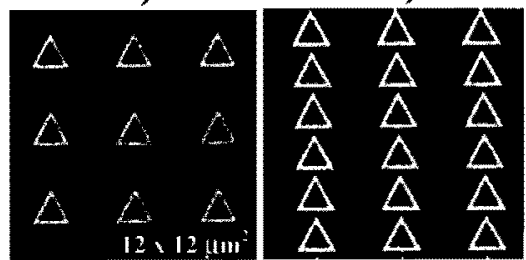

FIG. 1. SEM images of the three arrays of triangular rings investigated: (a) isolated rings, (b) vertical chains of rings, and (c) horizontal chains of rings. The size of the images is $12 \times 12 \mu \mathrm{m}^{2}$.

\section{EXPERIMENTAL DETAILS}

We have investigated three arrays of Py equilateral triangular rings (nominal side $2 \mu \mathrm{m}$, width $250 \mathrm{~nm}$, and thickness $25 \mathrm{~nm}$ ). The ring structures have been lithographically patterned on a 25-nm-thick Py film deposited onto a standard Si substrate followed by the lift-off step. The individual Py rings are ordered on the three different lattices shown in the scanning electron microscopy (SEM) images in Fig. 1: a rectangular array of isolated rings and vertical and horizontal chains of rings. In the first case the rings are arranged on a slightly rectangular lattice with a period of $\sim 4.2$ and $\sim 4.6 \mu \mathrm{m}$ along the vertical and horizontal directions of Fig. 1 (a), so that the interelement distance is about $2 \mu \mathrm{m}$ and the dipolar interaction between individual triangles is thus negligible. In the vertical chains [Fig. 1(b)] a comer of each triangle is in proximity of the center of the edge of the triangle above with interelement spacing of $50 \mathrm{~nm}$. In the horizontal chains the proximity is between the adjacent corners of the triangles [corner-to-corner distance is $50 \mathrm{~nm}$; Fig. $1(\mathrm{c})]$.

The magnetization reversal and the DW displacement have been monitored using a setup combining the longitudinal and diffraction magneto-optical Kerr effect ( $L$ - and D-MOKE, respectively $\left.{ }^{14,15}\right)$. The external field was applied in the sample plane (plane $x y$ ) using a quadrupolar electromagnet that allows us to apply simultaneously two mutually orthogonal fields: a vertical magnetic field $H_{y}$ perpendicular to the optical incidence plane ( $y$ direction) and a horizontal magnetic field $H_{x}$ parallel to both the incidence and sample planes ( $x$ direction). The light source utilized is a red-diode laser (wavelength $633 \mathrm{~nm}$ ) polarized in the plane of incidence ( $p$ - polarization). The L-MOKE setup described in detail in Ref. 14 is based on the modulation of polarization technique (photoelastic polarization modulator operated at $50 \mathrm{kHz}$ and photodiode signal detected using a lock-in amplifier locked either at 50 or $100 \mathrm{kHz}$ for detecting Kerr ellipticity or rotation, respectively) and is used to detect the magnetization component (normalized to saturation magnetization) parallel to $H_{x}$ from the reflected signal $\left[m_{x}(H)\right]$.

The experimental D-MOKE setup and the magnetic formfactor formalism used for the interpretation of the measured loops have been described in detail in Ref. 15. Briefly, in the D-MOKE measurements the changes in the sample magnetization lead to variations in the intensity (I) of diffracted beams, leaving their polarization state unchanged. This arrangement corresponds to the transverse MOKE geometry and is used to detect the magnetization component parallel to $H_{y}$ from the diffracted signal $\left[m_{y}(H)\right]$. As shown in Ref. 15, the variation $\Delta \mathrm{I}_{d}^{m}(n)$ of the intensity of the $n$th diffracted order beam as a function of the applied field $H$ measured in D-MOKE loops is

$$
\Delta \mathrm{I}_{d}^{m}(n) \alpha \operatorname{Re}\left[f_{d}^{m}(n)\right]+\mathrm{A}(n) \operatorname{Im}\left[f_{d}^{m}(n)\right],
$$

where $f_{d}^{m}(n)=\iint m_{y}(H) e^{i n(2 \pi / L) x} d s$ is called magnetic form factor of order $n$. In the previous expression, $m_{y}(\mathrm{H})$ is the component of the magnetization perpendicular to the scattering plane (plane $x z$; transverse MOKE geometry), $L$ is the array period in the $x$ direction parallel to the plane of incidence, $n$ is an integer, and the integral is carried out over the unit cell containing a single element of the array. Therefore, the D-MOKE loops contain information about the Fourier components of the magnetization and consequently provide information on the magnetization distribution within a unit cell of the array.

In our experiments these two different types of MOKE measurements are carried out simultaneously. In summary, for a given field sweep D-MOKE probes the component of $\boldsymbol{m}$ parallel to $y\left[m_{y}(H)\right]$ and L-MOKE the component along $x\left[m_{x}(H)\right]$.

The field dependence of the spin structure in the rings as well as the magnetic form factors are evaluated by calculating the magnetization distribution inside the particle at each applied field, using the micromagnetic OOMMF code ${ }^{16}$ In the case of the chains of rings we used a version of OOMMF modified to allow for periodic boundary conditions along the chain direction. ${ }^{17}$ This update enabled us to simulate a chain of interacting rings while actually considering a single ring in the simulations. The material parameters used for the simulations are those contained in the OOMMF program for permalloy (viz., saturation magnetization $M_{s}=800$ $\times 10^{3} \mathrm{~A} / \mathrm{m}$, exchange stiffness constant $A=1.3$ $\times 10^{-11} \mathrm{~J} / \mathrm{m}^{3}$, and no magnetocrystalline anisotropy has been considered; the damping coefficient used in the simulations is 0.5 ). Due to limited computational resources we used a cubic cell of $8.5 \mathrm{~nm}$ side, which is slightly larger than the exchange length of $\sim 5.7 \mathrm{~nm}$ defined as $\lambda_{\mathrm{ex}}=\sqrt{\left(2 A / \mu_{0} M_{s}^{2}\right)}$. In a few cases we have checked the results of simulations using a smaller cell of $5 \mathrm{~nm}$ side and we obtained similar results. Once the form factors are known at each field, the D-MOKE loops of any given order $(n)$ can be obtained from Eq. (1).

The arrays were also investigated by magnetic force microscopy (MFM). By suitably choosing the field history, various metastable states were quenched to zero field in the ring structures and subsequently imaged by MFM using an A. P. E. Research atomic force microscopy A50-CL tool.

Magnetic images were obtained using CoCr-coated MFM tips with typical scan heights of about $100 \mathrm{~nm}$ and the probe tip was magnetized to be a north pole.

\section{RESULTS AND DISCUSSION}

The magnetization reversal in isolated Py triangular rings [sample shown in Fig. 1(a)] has been the subject of a previous detailed investigation using D-MOKE combined with 
a)

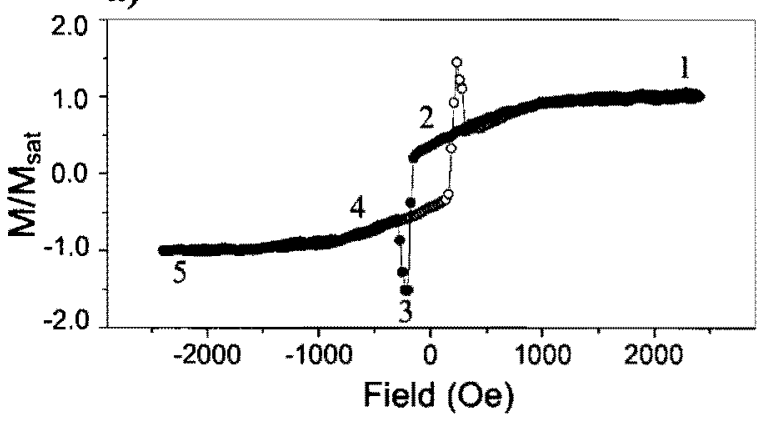

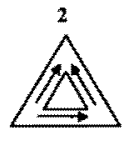
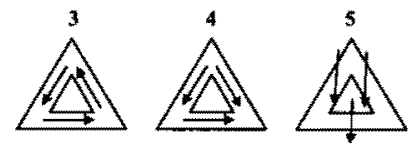

b)
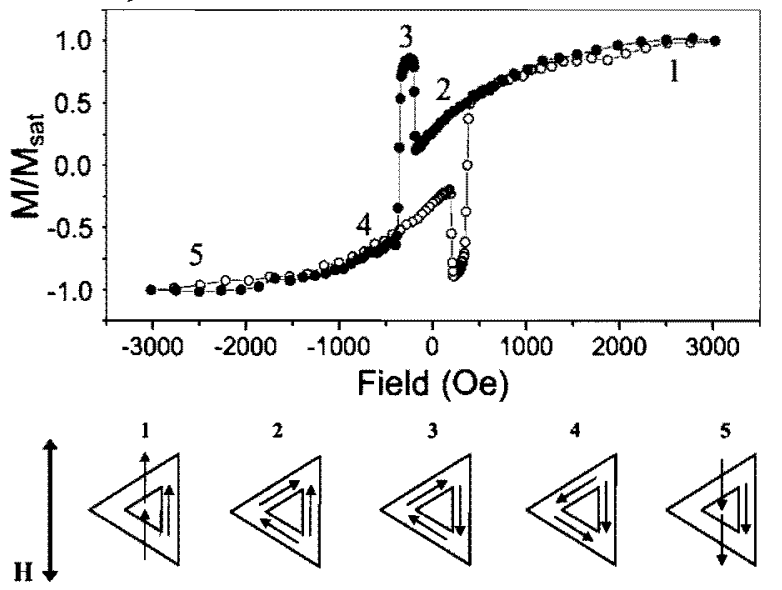

FIG. 2. (a) Second- and (b) first-order diffracted MOKE hysteresis loops from an array of isolated rings with the field perpendicular (a) and parallel (b) to an edge. The sketches below each loop are schematics of the magnetic configurations that are reached during reversal.

numerical micromagnetic simulations and in-field MFM." Figure 2 summarizes the results of that investigation (the loops in which the main features are most clearly discernible were selected): the upper panel (a) shows the second order D-MOKE loop recorded by applying the external field a few degrees ( 2 degrees clockwise) from perpendicular to an edge; the lower panel (b) displays the first-order D-MOKE measured with the external field applied parallel to an edge. For both panels we show sketches of the relevant magnetic configurations occurring during the reversal determined by micromagnetic simulations and confirmed by MFM imaging. They show that the switching occurs from an asymmetric onion state (sketch 2) to the reversed onion state (sketch 4) via a stable intermediate vortex state (sketch 3) for both directions of the applied field. The vortex state produces the intense peak in the D-MOKE loops. We note that an onion state is characterized by the presence of two DWs-one head to head and the other tail to tail-pinned, respectively, at two
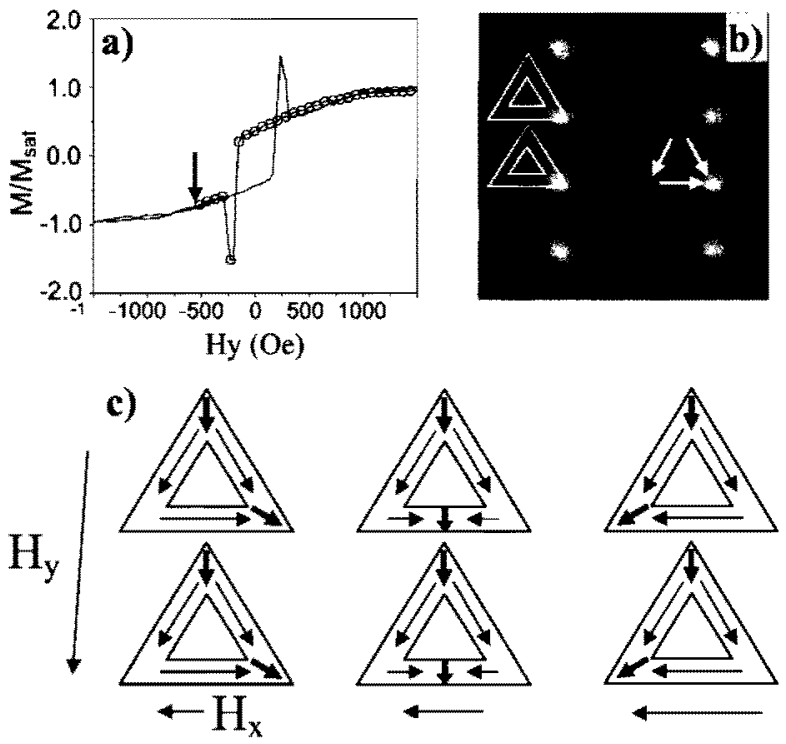

FIG. 3. (a) Second-order diffracted MOKE full (line) and minor (open dots) loops used to prepare the rings of the vertical chains in the same onion state. (b) Zero-field MFM image of the vertical chains showing the head-to-head and tail-to-tail DW that are present in the onion state. (c) Schematic diagrams showing the motion of a DW caused by a small horizontal field.

corners of the triangle that can be selected by a suitable field history (e.g., applying and removing the external field along a specific direction relative to the ring orientation).

\section{A. Vertical chains}

The D-MOKE loops measured from the sample with vertical chains of triangular rings, with the external field applied perpendicular to the edge $\left(H_{y}\right)$ of each ring that is closer to the corner of the nearest-neighboring ring, do not show appreciable differences with respect to those obtained from isolated rings shown in Fig. 2(a) (second-order diffracted loop). This means that the magnetization reversal of the rings arranged in vertical chains takes place via the same states as for the isolated rings, viz., from an onion state to the reversed state via the formation of a vortex. Based on this result we prepared all the rings, both in the isolated and in the chains samples, in a defined onion state by applying a positive saturating field $H_{y}$ and then reducing it to a welldefined negative value. This value has been selected by monitoring in real time the second-order D-MOKE loop and stopping the field sweep just after the peak corresponding to the vortex state as shown by the arrow in Fig. 3(a). Since once induced the onion state is stable at zero field, an MFM image was taken after the above process to verify that we had indeed prepared the sample in the desired state. The image taken from the chains of rings is presented in Fig. 3(b) and shows that indeed all the rings are in the same onion state with a DW (head-to-head type) in the bottom-right corner and another one (tail-to-tail type) in the upper corner. We thereafter induced the motion of the bottom-right DW and determined its pinning potentials by recording L-MOKE 

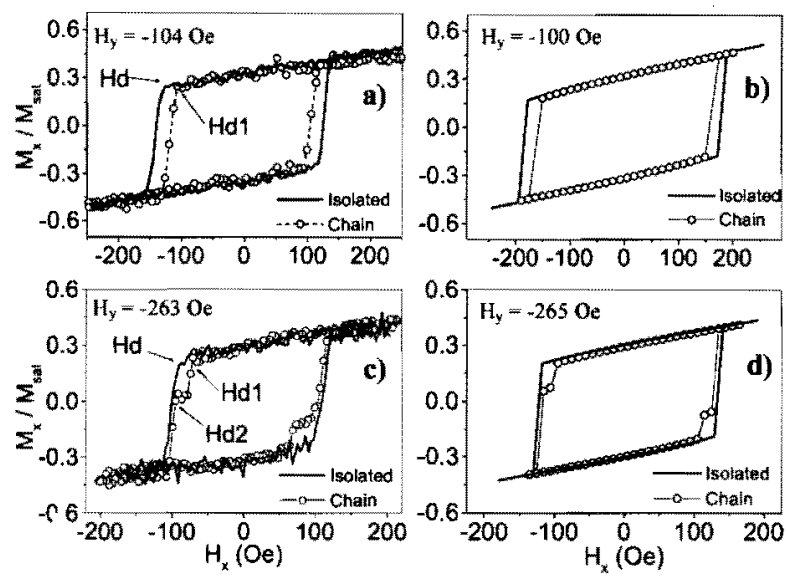

FIG. 4. [(a) and (c)] Measured and [(b) and (d)] simulated hysteresis loops for the DW motion indicated in Fig. 3(c). Hd and Hdl are the fields necessary to unpin the DW from the vertex in isolated and interacting triangles, respectively. $\mathrm{Hd} 2$ is the field necessary to unpin the DW from the potential well at the center of the base created by neighboring ring.

loops obtained by sweeping the wall with a second field $H_{x}$ applied along the bottom branch of the ring. We studied this process as a function of the strength of the final $H_{y}$ field used to prepare the initial onion state. This field prevents the oblique edges from switching, as sketched in Fig. 3(c).

Figure 4(a) shows the comparison between the L-MOKE loops recorded from the isolated and vertical chains rings for $H_{y}$ equal to -104 Oe. Both samples' loops display a single transition as the external field $H_{x}$ reaches a critical value $\mathrm{Hd}$ and $\mathrm{Hd} \mathrm{l}$ for the isolated and vertical chains sample, respectively. This means that the DW moves freely between the geometric pinning potential wells determined by adjacent corners as the external field $H_{x}$ reaches a critical value.

Quite interestingly, the depinning of the DW from the corners occurs at smaller fields in the chains with respect to isolated rings, viz. $|\mathrm{Hd} 1|<|\mathrm{Hd}|$. This is also confirmed by the loops calculated by micromagnetic simulations shown in Fig. 4(b). The reason for this is the presence of the additional potential well created at the center of the horizontal branch by the magnetostatic dipolar interaction between the rings in the chains.

The effects of the dipolar interaction in the chain of rings become more evident by further decreasing $H_{y}$. Figures 4(c) and 4(d) show the measured and calculated L-MOKE loops at $H_{y}$ equal to $-263 \mathrm{Oe}$. A single transition is still observed in the loops corresponding to the isolated rings but in the case of interacting rings the loops show an intermediate step caused by the pinning of the wall by the dipolar field emanating from the corner of the nearest ring neighbor. In this case the DW is released from the comer at $H_{x}=\mathrm{Hd} 1$ but it moves only to the center of the horizontal segment until $H_{x}$ reaches a second value $\mathrm{Hd} 2$ required to displace the $\mathrm{DW}$ completely to the adjacent comer. It is worth noticing that the drop in magnetization at the first jump in both the measured and calculated L-MOKE loops is smaller than that occurring at the second jump. This is somewhat surprising since the pinning is expected at the midpoint; we will show

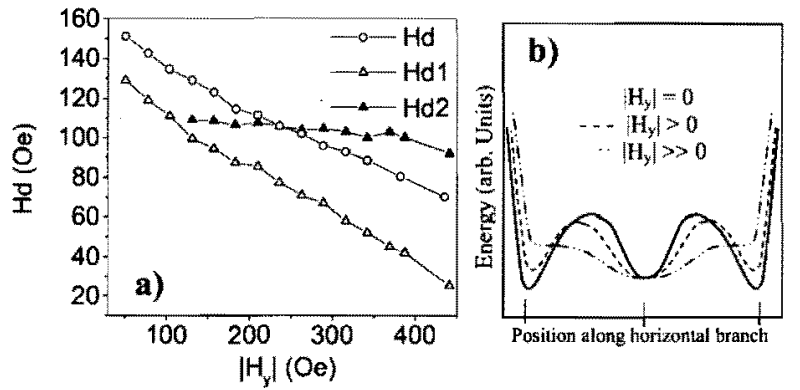

FIG. 5. (a) Evolution of $\mathrm{Hd}, \mathrm{Hdl}$, and $\mathrm{Hd} 2$ as a function of the field strength (absolute value) along $y,\left|H_{y}\right|$. (b) Schematic of the triple potential well and its evolution with $\left|H_{y}\right|$. The two side wells are a geometric effect of the vertices; the central well is caused by the neighboring ring.

later that this is due to the details of the actual spin configuration that sets up after the first partial displacement of the DW. In Fig. 5(a) the different values of $\mathrm{Hd}, \mathrm{Hdl}$, and $\mathrm{Hd} 2$ are plotted as a function of the absolute value of the amplitude of the field $\left|H_{3}\right|$ in the range $50-450 \mathrm{Oe}$. We observed that $H_{y}$ affects significantly the depinning field $\mathrm{Hdl}$ from the corner (open triangles) but to a lesser extent the depinning field $\mathrm{Hd} 2$ from the potential well created by the magnetostatic interaction [full triangles in Fig. 5(a)]. For higher values of $\left|H_{y}\right|$ (we explored a $\left|H_{y}\right|$ field range up to $700 \mathrm{Oe}$ ) both $\mathrm{Hd} 1$ and $\mathrm{Hd} 2$ are affected by $H_{y}$. This is due to a substantial change in the DW spatial extension at such higher values of $\left|H_{y}\right|$.

In the $\left|H_{y}\right|$ range up to $450 \mathrm{Oe}$, the process can be qualitatively modeled as a domain wall in a triple potential-well landscape that evolves as a function of the vertical applied field $H_{y}$ as sketched in Fig. 5(b) (the effect of $H_{x}$ on the potential landscape is not considered for simplicity). The basic idea can be summarized as follows: the vertical field $H_{y}$ modifies the energy landscape through which the domain wall has to move between the two corners by progressively twisting the spins in the horizontal segment toward the $-y$ direction. When $H_{y}$ is small the spins are nearly horizontal and the displacement of the domain wall is highly hindered, i.e., it costs more energy, as compared to the case in which they are twisted toward the $y$ axis, at higher negative values of $H_{y}$, so that the DW can move more easily along the ring segment. The effect of increasing $\left|H_{y}\right|$ corresponds, thus, to a progressive reduction in the energy barrier separating the two external potential wells of the corners. In the case of the isolated triangles where there are only the two potential wells due to geometrical pinning sites at the corners, the increase in $\left|H_{y}\right|$ leads to a reduction in Hd shown in Fig. 5(a).

In the case of interacting triangles, the behavior described above combines with the presence of the third potential well whose depth is essentially unaffected by $H_{y}$ and the following behavior can be envisioned: for small $\left|H_{y}\right|$ [solid line in Fig. 5(b) $]$ the value of $H_{x}$ required to unpin the DW from one corner is also sufficient to draw the domain wall through the middle well across the entire horizontal segment length so that a single transition is observed in the L-MOKE loop at a certain critical horizontal field Hd1; as $\left|H_{y}\right|$ increases above a 

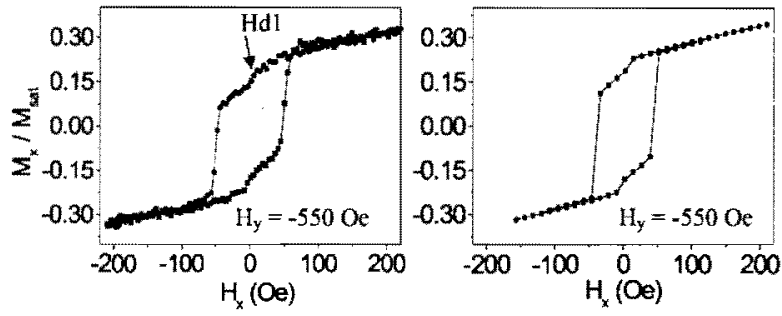

FIG. 6. Measured and simulated L-MOKE loops for $H_{y}=$ $-550 \mathrm{Oe}$ and for the vertical chains.

certain threshold [dashed line in Fig. 5(b)], the value of the horizontal field $\mathrm{Hdl}$ required to depin the domain wall from one comer is lower than the value $\mathrm{Hd} 2$ needed to unpin it from the central well so that once the wall has reached this well it moves no further until $H_{x}$ reaches $\mathrm{Hd} 2$. This results in the observation of two transitions in the L-MOKE loop. This simple picture qualitatively explains the measured and calculated L-MOKE loops. Based on this picture, there should be a value of $\left|H_{y}\right|$ at which the DW moves to the center of the horizontal segment even if $H_{z}$ is equal to zero [double dotdashed line in Fig. 5(b)]. For $\left|H_{y}\right|$ above this critical value the wall should move toward the center before $H_{x}$ has changed sign. This indeed is observed experimentally and is also reproduced by the simulations: the corresponding loops are shown in Fig. 6 where the measured and calculated L-MOKE loops for $H_{y}=-550$ Oe are displayed. We note that in this case we are already in the field range in which $\mathrm{Hd} 2$ is also affected by $H_{y}$ as discussed before.

To investigate the validity of the simple triple potentialwell picture we checked if the state with the DW pinned by the magnetostatic interaction at the midpoint of the horizontal segment is stable. We measured the minor L-MOKE loop shown in Fig. $7\left(\right.$ b) obtained with $H_{y}=-350$ Oe and by reducing $H_{x}$ to zero once the domain wall has been pinned at the center of the horizontal segment [the maximum intensity of $H_{x}$ falls in the plateau between the two steps in the L-MOKE loop shown in Fig. 7(a)]. The fact that the signal returns to its starting value indicates that the DW moves back to its initial position as $H_{x}$ is removed. It is important to note that the DW moves back despite of the presence of a small negative horizontal field component due to the $2^{\circ}$ tilt clockwise of $H_{y}$.

A closer look at the details of the simulated magnetization configurations in the horizontal segment during the wall displacement evidenced an additional effect neglected so far in our simplified picture. Figure 7(c) shows the magnetization configurations inside the bottom side of an interacting triangular ring calculated at $H_{y}=-350 \mathrm{Oe}$. In particular the picture shows the domain-wall pinning at the center during its displacement from the bottom-right corner of the ring to the center of the horizontal segment. The spin configuration is asymmetric with respect to the wall position: on the left-hand side the magnetization vectors are aligned along the same direction and pointing against the domain wall, while on the right side the spin distribution forms a so-called " $C$ " configuration. This asymmetry in the spins configuration gives rise to an "exchange" pressure $P$, sketched in Fig. $7(\mathrm{c})$, on the
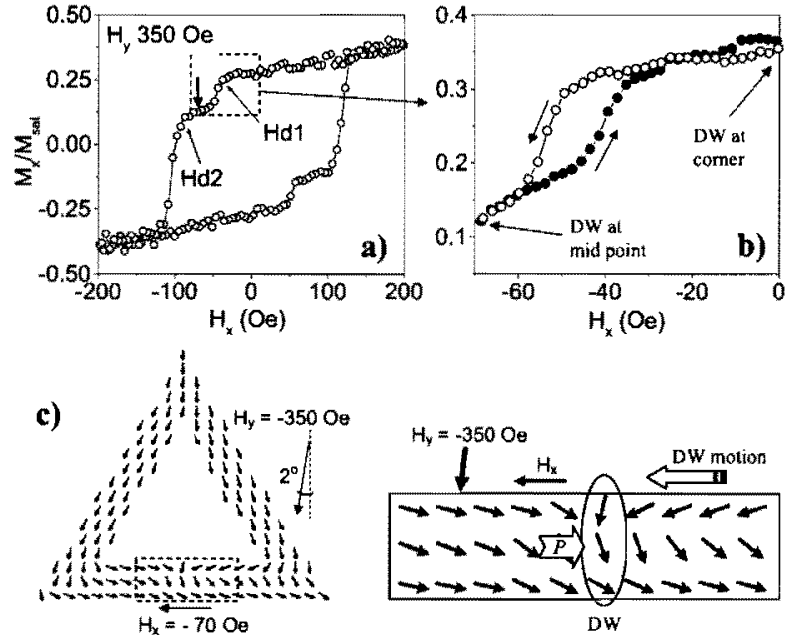

FIG. 7. (a) Full and (b) minor longitudinal MOKE loops for $H_{y}=-350 \mathrm{Oe}$ and for the vertical chains. The minor loop shows the reversibility of the DW motion. (c) Schematic of the spin distribution that leads to the reversible motion of the DW between one vertex and the center. The big arrow with the label " $P$ " indicates the exchange pressure determined by the asymmetric spin distribution at the two sides of the DW.

wall, which acts against the action of the external field. It is this asymmetry between the left- and right-hand side spin configurations that makes the intermediate state with the DW pinned by the magnetostatic interaction unstable so that if the field $H_{x}$ is removed the $\mathrm{DW}$ is pushed back to its original position by the "exchange" pressure.

This translates also into an off-center DW trapping region, i.e., the midpoint of the positions at which the DW is pinned by the magnetostatic interaction is slightly shifted toward the corner at which the DW originated.

The " $C$ " configuration of the spins in the right-hand side accounts also for the lower drop in magnetization during the first jump in both the measured and calculated L-MOKE loops as compared to that occurring in correspondence of the second jump mentioned above.

In summary, the simple triple potential-well picture described above does not explain why the DW is trapped off center in the case of interacting triangles as well as the reversibility observed in the minor loops. The reason for the model's shortcoming can be seen in the spin distribution obtained in micromagnetic simulations and shown in Fig. 7. While our model assumes a mirror symmetry around the triangle's midpoint, the micromagnetic spin distribution around a DW trapped in the "center" (Fig. 7) does not possess this symmetry. This means that the DW at the center has a memory of the corner at which it originated. With this insight, to treat the problem quantitatively in a potential-well framework, it would be necessary to consider a triple potential for a domain wall originating at the left (L) and another for those originating on the right (R). In this picture the DW motion induced by a transverse field would proceed as follows: at an initial critical field the DW would move to the off-center "central" well - the new potential would now account for the off-center trapping and for the reversibility. At 


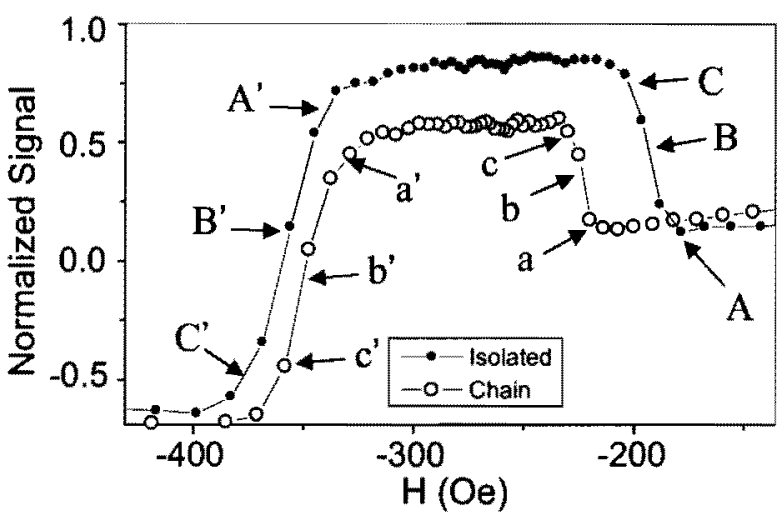

FIG. 8. Enlargement of one of the peaks in the first-order diffracted MOKE hysteresis loops for isolated rings (full dots) and horizontal chains (open dots) corresponding to the formation of the vortex state. The figure shows how the rings interaction changes the vortex nucleation field in the case of horizontal chains.

larger transverse fields the DW jumps from the L-DW into the R-DW landscape; the barrier in this case being primarily exchange energy.

Despite its shortcomings, the simple model presented above is useful because of the physical picture it provides about the DW trapping in the center as a function of the vertical applied field.

\section{B. Horizontal chains}

In the case of horizontal chains we expect appreciable effects of dipolar interaction when the external field is applied parallel to the chain direction. In this geometry alternated head-to-head and tail-to-tail DWs are formed very close to each other at the corners of adjacent rings along the chain. We therefore expect a strong dipolar stabilizing interaction between neighboring triangles. Figure 8 shows an expanded view of the descending branch of the first-order D-MOKE loops measured from the isolated and horizontal chains of rings. The mesalike peak corresponds to the formation of the intermediate vortex state occurring during the magnetization reversal in both ring arrangements [stage 3 in Fig. 2(b) $].{ }^{11}$ As can be seen in Fig. 8, the peak observed in the D-MOKE loop from the chains is significantly narrower than that measured on the isolated rings [the different intensity of the peaks has to be ascribed to the slightly different periods of the two arrays along the $y$ direction that affects the peak intensity as can be understood considering that it results in different values of the parameter $\mathrm{A}(n)$ in Eq. (1)]. In the first instance this result tells us that the dipolar interaction reduces the field range in which the intermediate vortex state exists by about $35 \mathrm{Oe}$. The reduction is due mainly to a delayed nucleation of the vortex configuration. This conclusion has been confirmed by micromagnetic simulations. The delayed nucleation can be easily understood considering that the attractive character of the dipolar interaction between the DWs in the chains increases the stability of the onion state preceding the formation of the vortex configuration, similarly to what is observed in interacting circular ${ }^{4-6}$ and elon-
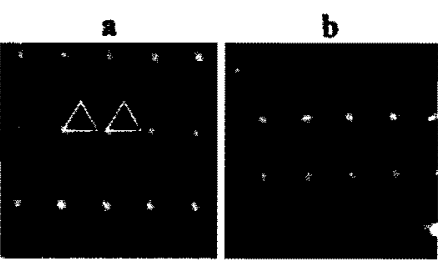

a
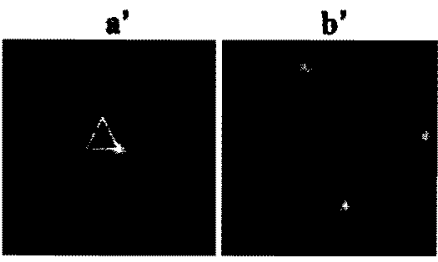

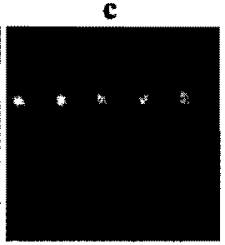

c'

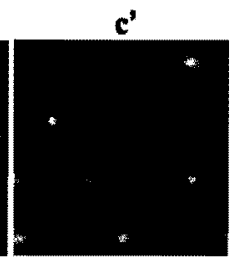

FIG. 9. MFM images corresponding to points labeled $a, b, c, a^{\prime}$, b', and $c^{\prime}$ in Fig. 8 and showing the correlated nucleation of the vortex state in horizontal chains $(a, b$, and $c)$ and the random nucleation of the onion state ( $a^{\prime}, b^{\prime}$, and $\left.c^{\prime}\right)$. The size of each image is about $10 \times 10 \mu \mathrm{m}^{2}$.

gated rings. ${ }^{7}$ Previous investigations showed also an anticipated annihilation of the vortex state due to a collective switching of the interacting rings induced by the switch of individual rings in a chain determined by ring's intrinsic properties. ${ }^{4-7}$ In Fig. 8 we observe a slightly anticipated annihilation of the vortex state but the effect in our case is extremely small and could be caused by a slightly different distribution of the vortex annihilation fields in the two samples. These differences can be attributed to the inevitable fabrication defects, such as deviation from the ideal shape, edge roughness, and material inhomogeneities. The fabrication defects also affect, to some extent, the vortex nucleation field.

Using MFM we have imaged the various magnetic configurations in the rings by quenching them to zero field. In Fig. 9 we show the distribution of vortex and onion states in the isolated and horizontal chains of rings after reducing to zero the external field from different points on the D-MOKE loop. Images $a, b$, and $c$ correspond to points labeled $a, b$, and $c$ in Fig. 8 and show the transition from the onion to the vortex state. Images $a^{\prime}, b^{\prime}$, and $c^{\prime}$ taken from samples quenched from the corresponding points in Fig. 8 show the vortex to onion transition. Figure 10 shows the equivalent images for the case of noninteracting rings. The images in Fig. 9 show clearly that during the transition from onion to vortex state (images $a, b$, and $c$ ) entire chains or long portions of chains of rings switch simultaneously, while during the transition from vortex to reversed onion state (images a', $b^{\prime}$, and $\left.c^{\prime}\right)$ the new onion states nucleate at random positions. During the first transition the attractive dipolar energy stabilizes the onion state well below the intrinsic (viz., determined by the ring geometry and fabrication defects) nucleation field so that when one ring switches to the vortex configuration the two nearest-neighbor rings become unstable and switch as well to the vortex configuration and so on in a sort of cascade process that might lead to the switch of an entire chain. This does not happen during the second transition since the dipolar interaction between the vortex states is negligible and the reversed onion states nucleation is 


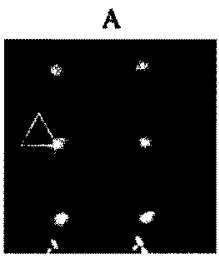

$A^{\prime}$
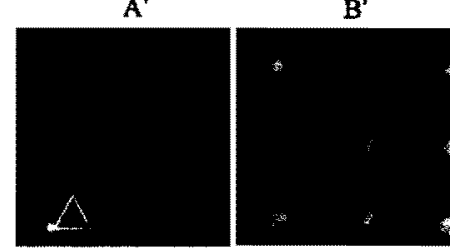

$B^{\prime}$
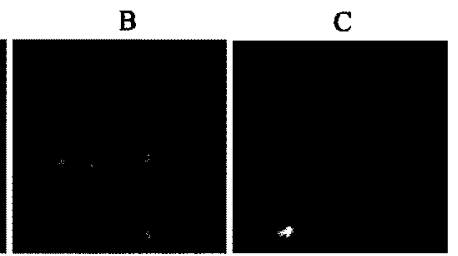

$\mathrm{C}^{\prime}$

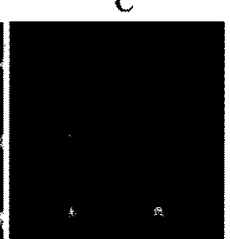

FIG. 10. Same as Fig. 9 but for isolated rings showing the uncorrelated nature of both vortex (A, B, and C) and onion state $\left(\mathrm{A}^{\prime}, \mathrm{B}^{\prime}\right.$, and $\left.\mathrm{C}^{\prime}\right)$ nucleation.

only determined by the intrinsic nucleation field distribution. This finding confirms that at variance with what previously reported on circular and elongated rings, ${ }^{4-8}$ in interacting triangular rings the annihilation of the vortex state is substantially unaffected by the dipolar interaction. In the case of isolated rings the dipolar interaction is always negligible so that the intrinsic nucleation field distribution governs both transitions and the vortex nucleation and annihilation processes occur at random positions in the sample as can be seen from the MFM images shown in Fig. 10.

\section{CONCLUSIONS}

We have investigated the effect of interelement interaction in arrays of triangular rings. Triangular rings are particularly interesting because the remanent state of each isolated ring is an "onion" state characterized by two domain walls-one head-to-head the other tail-to-tail-that form at the vertices of the triangle. When the rings are fabricated in close proximity the magnetic-dipole fields generated by these domain walls are shown to affect the switching of their neighbors. For head to base chains of triangles we show that the presence of a vertex close to the center of an edge can qualitatively change the manner in which reversal occurs by trapping the domain wall in the center of the base rather than at the vertices.

Based on the above, and clearly well beyond the scope of the present work, it is interesting to speculate that if one were to construct a triangular ring with two smaller triangular rings below its base, switching in the large ring might be tailored to depend on the magnetic state of both small rings. Such structures could lead directly to magnetic logic gates.

\section{ACKNOWLEDGMENTS}

V.M. acknowledges support by the U.S. NSF under Grant No. ECCS-0823813 and by the U. S. Department of Energy Office of Science Office of Basic Energy Sciences under Contract No. DE-AC02-06C1357 (CNM ANL Grants No. 468 and No. 470).
'P. Vavassori, V. Metlushko, and B. Ilic, Appl. Phys. Lett. 91, 093114 (2007).

${ }^{2}$ M. Kläui, C. A. F. Vaz, J. Rothman, J. A. C. Bland, W. Wernsdorfer, G. Faini, and E. Cambril, Phys. Rev. Lett. 90, 097202 (2003); M. Kläui, C. A. F. Vaz, J. A. C. Bland, W. Wernsdorfer, G. Faini, and E. Cambril, Appl. Phys. Lett. 81, 108 (2002).

${ }^{3}$ J. Rothman, M. Kläui, L. Lopez-Diaz, C. A. F. Vaz, A. Bleloch, J. A. C. Bland, Z. Cui, and R. Speaks, Phys. Rev. Lett, 86, 1098 (2001); M. Kläui, J. Rotthman, L. Lopez-Diaz, C. A. F. Vaz, J. A. C. Bland, and Z. Cui, Appl. Phys. Lett. 78, 3268 (2001).

${ }^{4}$ Xiaobin Zhu, P. Grütter, V. Metlushko, Y. Hao, F. J. Castaño, C. A. Ross, B. Ilic, and H. 1. Smith, J. Appl. Phys, 93, 8540 (2003).

${ }^{5}$ M. Kläui, C. A. F. vaz, J. A. C. Balnd, and L. J. Heyderman, Appl. Phys. Lett. 86, 032504 (2005).

${ }^{6}$ M. Kohda, K. Toyoda, T. Miyawaki, A. Fujita, and J. Nitta, J. Appl. Phys. 103, 07A714 (2008),

${ }^{7}$ J. Wang, A. O. Adeyeye, and N. Singh, Appl. Phys. Lett. 87, 262508 (2005).

${ }^{8}$ M. Laufenberg, D. Bedau, H. Ehrke, M. Kläui, U. Rüdiger, D. Backes, L. J. Heyderman, F, Nolting, C. A. F. Vaz, J. A. C. Bland, T. Kasama, R. E, Dunin-Borkowski, S. Cherifi, A. Loca- telli, and S. Heun, Appl. Phys. Lett. 88, 212510 (2006).

${ }^{9}$ D. A. Allwood, G. Xiong, C. C. Faulkner, D. Atkinson, D. Petit, and R. P. Cowburn, Science 309, 1688 (2005).

${ }^{10} \mathrm{P}$. Vavassori, M. Grimsditch, V. Novosad, V. Metlushko, and B. Illic, Phys. Rev. B 67, 134429 (2003); A. Libá, M. Grimsditch, V. Metlushko, P. Vavassori, and B. Janko, J. Appl. Phys. 98. 083904 (2005).

${ }^{11}$ P. Vavassori, O. Donzelli, M. Grimsditch, V. Metlushko, and B. Ilic, J. Appl. Phys. 101, 023902 (2007).

${ }^{12}$ A. Westphalen. A. Schumann, A. Remhof, H. Zabel, T. Last, and U. Kunze, Phys. Rev. B 74, 104417 (2006).

${ }^{13}$ A. Imre, G. Csaba, L. Ji, A. Orlov, G. H. Bernstein, and W. Porod, Science 311, 205 (2006).

${ }^{14}$ P. Vavassori, Appl. Phys. Lett. 77, $1605(2000)$.

${ }^{15} \mathrm{M}$. Grimsditch and P. Vavassori, J. Phys.: Condens. Matter 16, R275 (2004).

${ }^{16}$ M. J. Donahue and D. G. Porter, OOMMF User's Guide, Release 1.2a4pre, National Institute of Standards and Technology Report No. NISTIR 6376, 1999.

${ }^{17}$ K. M. Lebecki, M. J. Donahue, and M. W. Gutowski, J. Phys. D: Appl, Phys. 41, 175005 (2008). 